\title{
Converse Theory of the Binomial Theorem.
}

By Mr Sita noth Chokrobarthy.

\section{[Abstract.]}

The method of extracting any root may be understood from the subjoined example of extracting the fifth root of $2,073,071,593$.

$$
\begin{aligned}
& (x+y)^{5}=x^{5}+5 x \times{ }^{4} y+10 x^{3} y^{2}+10 \times{ }^{2} y^{3}+5 \times y^{4}+y^{5} . \\
& \overline{20730} \overline{71593}(73=\text { required root. } \\
& 7^{5}=16807 \\
& \left.5.7^{4}=12005\right) \overline{39237}(3 \\
& 5 \cdot 7^{4} \cdot 3=\frac{36015}{32221} \\
& 10 \cdot 7^{3} \cdot 3^{2}=\quad \frac{30870}{13515} \\
& 10 \cdot 7^{2} \cdot 3^{3}=\quad \frac{13230}{2859} \\
& 5.7 .3^{4}=\quad \frac{2835}{243} \\
& 3^{5}=\quad \frac{243}{0}
\end{aligned}
$$

Note: The given number is divided into periods of five digits beginning from the place of unity. The fifth power of the greatest number possible (7) is subtracted from the left hand period. This number is the first digit of the root required. To the remainder (3923) the first figure of the next period (7) is affixed. This number is then divided by five times the fourth power of the first digit of the root and the quotient is the second digit of the root. The formation of the rarious divisors following is obvious from the expansion of $(x+y)^{6}$. 